\title{
La teología práctica de Karl Rahner: Una teología pastoral en perspectiva escatológica
}

\author{
Alex Vigueras \\ FACULDADE JESUÍTA DE FILOSOFIA E TEOLOGIA, BELO HORIZONTE, BRASIL
}

\section{Introducción}

Este estudio es parte del trabajo de investigación de nuestra tesis de doctorado que tiene como título: «En la meta, hacia la meta: la perspectiva escatológica como horizonte de comprensión de la teología práctica en Karl Rahner», presentada recientemente para evaluación. Nuestra entrada en la teología práctica de Rahner comienza en el trabajo para la dissertação de mestrado, en la cual investigamos la concepción de teología práctica en Rahner y el contexto de los problemas a los cuales Rahner intentó responder. El título de esa dissertação es: «¿Qué tiene que hacer la Iglesia hoy? La concepción de teología práctica en Karl Rahner» .

Nuestro trabajo actual, entonces, está fundado en la convicción de que la teología práctica de Rahner tiene una fundamentación que es escatológica y, justamente allí radica su mayor riqueza y, paradójicamente, ha sido un ámbito poco explorado hasta ahora. Es solo en relecturas muy recientes que constatamos el interés por la dimensión escatológica de su teología práctica.

Por los límites que impone una presentación como esta no entraremos en el detalle de la problemática que está en el trasfondo de la propuesta de Rahner. Solo enunciaremos aquellas problemáticas que nos parecen más relevantes. La propuesta de Rahner se coloca críticamente delante de teologías pastorales que no tienen capacidad de ser verdaderamente críticas. Y esto ocurre porque, o bien han renunciado a su dimensión teórica,

\footnotetext{
A. Vigueras, ¿Qué tiene que hacer la Iglesia hoy? La concepción de teología práctica en Karl Rabner (Belo Horizonte 1999).
} 
transformándose en meros recetarios para la praxis del clero, una praxis ramplona, concebida como mera funcionalidad al servicio de los que ejercen el ministerio pastoral, o bien se conciben como meras mediadoras entre teoría y praxis, por cuanto les corresponde mediar la aplicación de los principios generales de la teología dogmática, a partir de un proceso meramente deductivo. A menudo esto ha tenido como consecuencia teologías pastorales que, simplemente, reafirman la praxis existente o, peor todavía, se alían a intereses ideológicos vigentes. En la base de estos problemas está un déficit en la fundamentación propiamente teológica de la teología pastoral, que la ha llevado muchas veces a doblegarse al rayado de cancha que le han planteado las otras ciencias. Por otro lado, Rahner reacciona delante de una pastoral que se concibe como ciencia al servicio del pastor, del clero, dejando en segundo plano la dimensión ministerial de todo bautizado.

En esta presentación nos detenemos, en primer lugar, en la concepción de teología práctica de Rahner, luego intentamos mostrar que su propuesta es la de una teología práctica en perspectiva escatológica para, finalmente, proponer algunas mediaciones posibles para un método de teología pastoral.

\section{La concepción de teología práctica en Karl Rabner}

La propuesta de Rahner que presentamos es el eje articulador del Manual de Teología Pastoral ${ }^{2}$, obra que nació por una serie de motivaciones convergentes $^{3}$ : en primer lugar, los significativos cambios socioculturales que adquieren un carácter mundial y que tienen un significado fundamental para la acción de la Iglesia. Se hace necesario un nuevo lenguaje que sea significativo para las personas en ese momento histórico concreto; en segundo lugar, la nueva autocomprensión de la Iglesia emanada del Concilio Vaticano II; y, en tercer lugar, la necesidad de una estrategia global de la Iglesia para responder a los nuevos desafíos, que supere la simple táctica pastoral del párroco considerado individualmente, y que supere también

2 K. Rahner; F. Arnold; V. Schurr; L. Weber (Hg.) Handbuch der Pastoraltheologie. Praktische Theologie der Kirche in ibrer Gegenwart, I - III (Freiburg I, 21970; II/1, ${ }^{21971 ; ~ I I / 2, ~}$ ${ }^{2}$ 1971; III, ${ }^{2}$ 1970); K. Rahner; V. Schurr; L. Weber; F. Klostermann (eds.) Handbuch der Pastoraltheologie IV (Freiburg 1969). Y como complemento: K. RAHNER; F. KLOStermann; H. Schild (Hg.) Lexikon der Pastoraltheologie. (Freiburg 1972). [En adelante: Handbuch].

3 Cf. K. Rahner et al., «Vorwort zum der HPth I» en $S W 19,535$. 
una pastoral caracterizada por la improvisación. Es un verdadero esfuerzo científico que no tiene que ver con una popularización barata de la ciencia, sino con la introducción en un pensamiento críticamente científico ${ }^{4}$. Toda esta obra es un esfuerzo por responder a la pregunta ¿qué tiene que hacer la Iglesia hoy? ${ }^{5}$. El Handbuch no quiere ser solo un trabajo de reflexión, sino un servicio a la praxis concreta de la Iglesia.

Dividimos nuestra presentación en cinco momentos: el objeto de la teología práctica, el análisis teológico de la situación presente, la finalidad de la teología práctica, sus relaciones con otras ciencias y, finalmente, su cientificidad.

\subsection{El objeto de la teología práctica}

La teología práctica es para Rahner «aquella ciencia teológica (es decir, sacada de las fuentes de la revelación, magisterialmente normada, que procede según un método, construida sistemáticamente y que utiliza los conocimientos profanos como cualquier otra disciplina teológica) que bajo el análisis cientifico y, precisamente, teológico de la situación concreta (y no contemplada aún adecuadamente en el aspecto jurídico) en-cada-momento de la Iglesia, desarrolla los principios (y en la medida de lo posible los concreta en imperativos), según los cuales la Iglesia en esta determinada situación (es decir, en-cada-momento actual) actualiza su propia esencia y así realiza su actividad de salvación ${ }^{6}$.

\subsubsection{Objeto material}

La teología práctica tiene como su objeto material la autorrealización de la Iglesia en su totalidad o, como está expresado arriba, «la actualización de su propia esencia». Esto incluye, por un lado, las distintas dimensiones en las cuales la Iglesia se autorrealiza (por ejemplo: el culto litúrgico, el anuncio de la Palabra, los sacramentos, la catequesis, la vida eclesial del individuo); y, por otro lado, los diferentes sujetos de esta autorrealización: ministros ordenados, laicos, comunidad local, grupos eclesiales, Iglesia en

4 Cf. K. H. Neufeld, «Genese und geschichtliche Hintergründe des, Handbuchs für Pastoraltheologie‘», PthI 24 (2/2004) 45-52; 50. [En adelante: Genese des Handbuchs].

5 K. RAHNER et al., Vorwort zum der HPth I, 535.

6 K. Rahner, «Pastoraltheologie- ein Überblick», SW 19, 3-29; 6-7. [Cursivas del autor]. 
general, etc. ${ }^{7}$ Dicho sintéticamente: «El objeto de la teología práctica son todos y todo en la Iglesia, es decir, todos los que son sujetos de la autorrealización de la Iglesia (...) Su objeto es todo; es decir, la autorrealización de la Iglesia en todas sus dimensiones» ${ }^{8}$.

\subsubsection{Objeto formal}

Ahora bien, la teología práctica tiene como objeto material esta autorrealización de la Iglesia en todas sus dimensiones, pero en la medida en que es considerada en el encuentro con la situación presente. Los adjetivos utilizados por Rahner para esta situación presente: «concreta...en-cada-momento», «determinada (es decir, en-cada-momento actual)», quieren advertir contra toda posibilidad de abstracción de esa situación presente, por cuanto es una situación siempre única e irrepetible o, mejor, en cada momento actual. La Iglesia, en cuanto que tiene que realizarse aquí y ahora o, dicho de otro modo, la condicionalidad de la realización de la Iglesia a través de la situación presente, es el punto de vista formal de esta disciplina. La teología práctica se preocupa de los principios de la autorrealización de la Iglesia, emanados de la dogmática, la teología moral y el derecho canónico, pero en cuanto los comprende en su actualización y concretización en la situación presente de la Iglesia ${ }^{9}$. Así ella no se desentiende de la dimensión esencial de la Iglesia, pues solo es posible saber lo que la Iglesia debe hacer en la medida que sabemos lo que ella es; no se trata de un saber genérico de lo que debe hacer siempre, sino lo que debe hacer aquí y ahora ${ }^{10}$.

La Iglesia es una institución histórica que no existe simplemente en una forma de concretización siempre igual. Ella debe acontecer en la historia de manera siempre nueva. Explicando la densidad teológica que tiene este acontecer histórico de la Iglesia, Rahner señala: «Su evento no es simplemente la presencia duradera de su esencia en una temporalidad y espacialidad que permanece externa a ella, sino la forma histórica de su esencia, que se da solo una vez, y que está destinada para ella por el Espíritu de la Iglesia a través de su situación histórica que se da solo una vez» ${ }^{11}$.

Cf. K. RAHNER, «Pastoraltheologie- ein Überblick», 6.8.

8 K. RAHner, «Die praktische Theologie im Ganzen der theologischen Disziplinen», $S W$ 19, 503-515; 506. [En adelante: «Praktische Theologie im Ganzen»].

9 Cf. K. RAHNER, «Pastoraltheologie- ein Überblick», 8.

10 Cf. K. Rahner, «Praktische Theologie im Ganzen», 505.

11 K. Rahner, «Praktische Theologie im Ganzen», 505. 
El hecho de ser la Iglesia de Cristo, sacramento de su victoria escatológica, no anula la tarea propia que ella tiene en la historia, la responsabilidad de su libertad, la capacidad de tomar decisiones y, por tanto, la posibilidad de cambio. La situación presente tiene para la Iglesia un significado teológico, como llamado inmediato de Dios, del cual la Iglesia no puede desentenderse; esta situación es el horizonte histórico fundamental donde Dios se autocomunica libremente a la persona humana; es un momento interno en la acción libre de la Iglesia, en su capacidad de decisión; es situación de la Iglesia misma, la cual es querida y dada a ella por Dios. Esta situación presente solo es comprendida y valorada correctamente en el trasfondo de la voluntad de Dios, y no como mero horizonte neutro.

\subsection{El análisis teológico de la situación presente}

Para la tematización de su objeto específico, la Teología Práctica recurre al análisis teológico de la situación presente. Es interesante la insistencia que Rahner hace en la definición presentada más arriba de la teologicidad como cualidad del análisis científico: «...bajo el análisis científico, y precisamente teológico...». Tal análisis es necesario por varias razones: en primer lugar, porque la teología práctica no puede tomar un análisis de este tipo de alguna de las ciencias que versan sobre la esencia, pues ellas no tienen medios para llevarlo a cabo. En segundo lugar, para evitar que la teología práctica sea un mero recetario práctico para la praxis pastoral, sin capacidad de situarse críticamente frente a esta; $y$, en tercer lugar, para evitar un análisis de la situación sin seriedad científica ${ }^{12}$.

El análisis de la situación presente debe ser teológico, pues solo un tal análisis atiende al ser complejo de la realidad presente como realidad teologal, como presente que es dato de la historia de la salvación de la Iglesia misma. El teólogo puede y debe ayudarse de las ciencias profanas para realizar este análisis, pero tiene que integrar estos conocimientos en una síntesis teológica ${ }^{13}$. Entonces, el análisis de la situación presente al que recurre la teología práctica, no debe entenderse como una ciencia auxiliar que prepara la reflexión propiamente teológica, sino como momento interno, pues tal reflexión debe clarificar teológicamente el presente.

12 Cf. K. RAHner, Strukturwandel der Kirche als Chance und Aufgabe (Freiburg 21972), 21-22. [En adelante: Strukturwandel der Kirche].

13 Cf. K. RAHnER, Strukturwandel der Kirche, 21. 
Este análisis debe prestar atención a la situación interna de la Iglesia y, al mismo tiempo a la situación del mundo, clarificando de qué modo esta situación está ordenada a la realización del ser histórico de la Iglesia, sea como condición de posibilidad, sea como contradicción con esta realización $n^{14}$.

\subsection{La finalidad de la teología práctica}

La teología práctica tiene como finalidad la planificación de la autorrealización de la Iglesia para el presente y el futuro. Esta planificación es distinta de la simple táctica pastoral que no se sitúa en un contexto global de la Iglesia, sino que está orientada a la praxis concreta y específica del clero solamente. No se trata de actualizar una determinada forma de vida eclesial que reemplace la actual, sino el posibilitar la reacción de la Iglesia de cara a las exigencias que la situación presente plantea. Una tal planificación que ayude a establecer prioridades en la acción de la Iglesia es necesaria, pues la estrategia salvífica de Dios, cuya gracia es infinita, no es igual a la de la Iglesia que cuenta con medios limitados y fuerzas finitas. Otra exigencia de esta planificación es que debe hacerse a tiempo, cuando se dan todavía las condiciones y posibilidades para llevar adelante un cambio ${ }^{15}$.

Ahora bien, en vistas a esta planificación, no es posible determinar el modo como la Iglesia debe actuar en la situación presente, a partir solo de los principios esenciales de la fe y mediante un proceso simplemente deductivo. Las normas concretas del actuar «no pueden ser, ni son, simplemente y siempre meras deducciones lógicas de estos principios de la fe, sino que siempre llevan en sí la irrepetibilidad de lo histórico concreto y del acto libre que las establece» ${ }^{16}$. Por ello, a partir del análisis teológico de la situación presente, la teología práctica «desarrolla los principios (y en la medida de lo posible los concreta en imperativos) ${ }^{17}$ para la planificación de la estrategia global de la Iglesia, los cuales no tienen el carácter de nor-

\footnotetext{
14 Cf. K. RAHNER, «Pastoraltheologie- ein Überblick», 8.

15 Cf. K. RAHner, Strukturwandel der Kirche, 55-56.

16 K. RAHner, Strukturwandel der Kirche, 14.

17 K. RAHNER, «Pastoraltheologie- ein Überblick», 6-7. Es extraño ese "y en la medida de lo posible" de esta definición, pues tenemos la impresión de que en el conjunto del planteamiento de la teología práctica en Rahner se expresa una necesidad de llegar a imperativos concretos. Esa concretud de los imperativos es colocada como distintivo de la teología práctica en relación con las ciencias teológicas que versan
} 
mas y leyes absolutas, sino que son propiamente una oferta al Pueblo de Dios y, especialmente, a los sujetos de la misión ${ }^{18}$.

Por esta naturaleza particular de los imperativos, la estrategia global de la Iglesia debe tomar distancia, tanto de las utopías intramundanas, que llegan a conclusiones de modo totalmente lógico y racional, cuanto de aquella forma de ciencia de planificación eclesial que quiere planificar todo en un pastoralismo apriorístico que cree poder prescindir de la situación presente de la Iglesia ${ }^{19}$.

La teología práctica ofrece a la Iglesia estos imperativos como orientación de su estrategia global, pero no le corresponde a esta disciplina la actualización de tales imperativos. La toma de decisiones corresponde a las instancias que tienen en la Iglesia un rol de gobierno. Dicho de otro modo, a la teología práctica le toca la investigación que tiene como resultados los imperativos y al gobierno eclesial la implementación de la estrategia global inspirada en ellos ${ }^{20}$.

\subsection{La relación con las otras ciencias}

En la definición que colocamos al inicio Rahner señala, antes de lo diferencial, aquello que asemeja a la teología práctica con las otras ciencias teológicas, por cuanto también es «sacada de las fuentes de la revelación, magisterialmente normada, que procede metódicamente, construida sistemáticamente y que utiliza los conocimientos profanos como cualquier otra disciplina teológica» ${ }^{21}$. Por el lado de la teologicidad subraya la vinculación necesaria con la revelación y el magisterio y, por el lado de la cientificidad, su proceder metódico y sistematicidad.

Una vez establecida la semejanza es posible señalar aquello que las diferencia, pero a partir de la relación. Es importante señalar que en la cla-

sobre la esencia. En todo caso es claro que los principios generales no quedan de ningún modo excluidos.

18 Cf. K. Rahner, «Stichworte aus dem Lexikon der Pastoraltheologie: Pastoraltheologie», SW 19, 489-493; 491. [En adelante: «Pastoraltheologie. Lexikon»].

19 H. Schuster, «Wesen und Aufgabe der Pastoraltheologie als praktischer Theologie», HPth I, 93-120;105. Tomamos como referencia a H. Schuster porque su concepción de Teología Práctica expresada en este primer volumen del Handbuch está en sintonía con la de Rahner.

20 Cf. K. Rahner, «Pastoraltheologie- ein Überblick», 15.

21 K. RAHNER, «Pastoraltheologie- ein Überblick», 6. 
rificación de la relación de la teología práctica con las ciencias en general y con las otras ciencias teológicas en particular, está en juego su carácter de ciencia propiamente tal, en el sentido de que tiene un objeto material y formal propio, distinto de las otras ciencias. La relación puede darse de los siguientes $\operatorname{modos}^{22}$ :

a) Como el todo se relaciona con sus partes: es la relación, por ejemplo, con la homilética, la catequética, la misionología.

b) Como una ciencia con la otra que, a pesar de examinar los mismos objetos materiales, lo hace en un nivel distinto de actualización y concretización: es la relación existente con las ciencias que versan sobre la esencia [Wesenswissenschaften] (la dogmática en general y la eclesiología dogmática en particular), que examinan la autorrealización de la Iglesia solo a partir de su ser abstracto. La teología práctica, en cambio, examina esta autorrealización en la situación presente. Estas disciplinas teológicas que versan sobre la esencia son fundamento, supuesto, horizonte y patrón de la teología práctica. Por su parte, la teología práctica hace notar a ellas que la tarea que les corresponde no es a-histórica o historicista (en el sentido de que no se plantean la pregunta por el anuncio hoy) y, que sus conclusiones no son una sabiduría siempre válida en el tiempo, sino que deben realizarse en una situación concreta de mundo y de la Iglesia; deben ser actualizadas en su globalidad ${ }^{23}$.

c) Como una ciencia con otra que tiene objetos materiales y fuentes de conocimiento y normas diferentes: es la relación existente entre teología práctica y las ciencias profanas.

d) Como una ciencia con las otras que examinan los mismos objetos (materiales y formales) en otra fase de tiempo, a saber, que examinan el pasado en lugar del presente y el futuro: es la relación existente entre teología práctica y la historia de la Iglesia, en la medida que esta última es la historia de la salvación pasada de la Iglesia. La teología práctica, en cambio, abarca el presente y el futuro; ella debe señalar a la historia de la Iglesia que no puede ser la mera curiosidad histórica, sino «el constituirse crítico de la memoria del pasado de la Iglesia» ${ }^{24}$. La historia de la Iglesia podría reconocer este pasado como manifesta-

22 Cf. K. RAHNER, «Pastoraltheologie- ein Überblick», 18-20.

23 Cf. K. Rahner, «Praktische Theologie im Ganzen», 511.

24 K. Rahner, «Praktische Theologie im Ganzen», 509. 
ción de posibilidades futuras y tareas de la Iglesia, en la medida que se interesa por preguntas teológicas auténticas y mira el pasado a partir de ellas; Rahner sintetiza: «Lo que cargó la Iglesia tras de sí es en definitiva interesante solo a partir de aquello que la Iglesia tiene todavía por delante $\rangle^{25}$.

Finalmente, la teología práctica debe hacer dos exigencias a las otras disciplinas teológicas ${ }^{26}$. en primer lugar, ella exige el reconocimiento de su originalidad y significado como una disciplina teológica propiamente tal; en segundo lugar, las otras disciplinas teológicas deben preservar y reconocer el momento de teología práctica que está y debe estar presente en ellas mismas, y esto porque todas las disciplinas teológicas deben estar al servicio de la autorrealización de la Iglesia. Rahner va todavía más lejos al plantear la teología práctica como principio organizativo de la teología en general: «Cuando se reconoce a la razón práctica (...) una prioridad, porque y en la medida que ella es la presencia-a-sí reflexionada de la realización de aquella acción que significa salvación, (...) entonces se le puede conceder a la teología práctica, como a la representante de la autorreflexión de esta razón práctica en la Iglesia, una prioridad en la teología como totalidad $»^{27}$.

\subsection{La cientificidad de la teología práctica}

Pese a que varios de los elementos que analizaremos a continuación ya han sido tratados, nos parece importante dar especial atención a la cientificidad de la teología práctica en la concepción de Rahner. La teología práctica es ciencia propiamente dicha porque tiene un objeto material propio: la autorrealización de la Iglesia; y un objeto formal propio: la autorrealización de la Iglesia en el presente y el futuro. La reflexión que ella realiza de modo científico-sistemático es de naturaleza inderivable en cuanto es una reflexión para una decisión. Ninguna de las otras ciencias teológicas puede realizar esta tarea con los medios de que dispone.

Ella tiene un significado práctico porque, a partir del análisis de la praxis de autorrealización de la Iglesia, así como se da en el presente, elabora los imperativos para la estrategia global de la Iglesia que debe orientar la praxis de cada Iglesia particular. Dada esta cercanía con la praxis

\footnotetext{
25 K. Rahner, «Praktische Theologie im Ganzen», 513. [Cursivas del autor].

26 Cf. K. Rahner, «Praktische Theologie im Ganzen», 508.

27 K. RAHNER, «Pastoraltheologie. Lexikon», 492-493.
} 
eclesial, Rahner afirma que no es la Universidad el único lugar donde puede hacerse teología práctica, pues ella debe estar presente en la trama viva de la vida eclesial. «Porque la teología práctica tiene como objetivo la acción de la Iglesia y porque en definitiva solo en la acción misma se da la última autocerteza respecto a la verdad de la acción para ella misma, la teología práctica nunca puede ser realizada solo como 'universitaria'. Ella no solo tiene que estar referida a la Iglesia, a su ministerio, sino que también debe provenir de ahí» ${ }^{28}$.

La teología práctica es teoría también cuando es un momento de la praxis de la Iglesia; pero no es teoría como lo son las ciencias que versan sobre la esencia, sino de un modo totalmente propio como reflexión direccionada a [una] decisión ${ }^{29}$. Esto implica un elemento profético y uno político. Esta ciencia teológica es teoría en el sentido más auténtico «porque también la teoría realizada realmente es un momento interno de la praxis de la Iglesia y solo bajo este presupuesto, permanece auténtica teoría ${ }^{30}$.

La teología práctica tiene una función crítica en dos direcciones ${ }^{31}$ : en relación con la praxis de la Iglesia, ayudándola a superar su autorrealización así como está dada y que es siempre deficiente; y en relación con las otras disciplinas teológicas, pues les pregunta si contribuyen suficientemente para una decisión real para la autorrealización de la Iglesia, desempañando así un rol hermenéutico ${ }^{32}$.

Finalmente, la teología práctica es ciencia porque tiene un método propio, determinado por dos momentos: el primero es el análisis científico-teológico de la situación presente y, el segundo, la definición de los imperativos para la estrategia global de la autorrealización de la Iglesia en el presente y en el futuro.

\section{Una teología práctica en perspectiva escatológica: recepción crítica y fundamentación}

Nos parece distinguir dos momentos en la recepción crítica del concepto de teología práctica en Rahner en el ámbito de los teólogos de habla alemana. Situamos la primera recepción en el tiempo que va desde la pu-

\footnotetext{
28 K. RAHNER, «Pastoraltheologie. Lexikon», 491.

29 K. RAHnER, «Praktische Theologie im Ganzen», 505.

30 K. Rahner, «Praktische Theologie im Ganzen», 506.

31 Cf. K. RAhner, «Praktische Theologie im Ganzen», 506.

32 Cf. M. Midali, Teologia pastorale o pratica: Cammino storico di uma riflesione fondante e scientifica (Roma 1985) 166.
} 
blicación del Handbuch, hasta el año 1980. Percibimos como un hito de esta primera recepción, el Congreso realizado en Viena en enero de 1974, organizado por la Conferencia de Teólogos Pastorales de lengua alemana, con el título: Teología Práctica 1774-1974. En esa ocasión se conmemoraban los 200 años del nacimiento de la teología práctica como ciencia teológica universitaria, cuyo inicio se establece en 1774 con la publicación del Esbozo de una mejor organización de las escuelas de teología de Stephan Rautenstrauch $^{33}$. De modo general, se valora el aporte realizado por Rahner en el Handbuch, pero se considera ya sobrepasado. La publicación que reunió las ponencias de ese encuentro, titulada Pastoral Theologie heute, contiene planteamientos críticos de varios autores ${ }^{34}$. Estimamos que esta primera recepción se prolonga hasta 1980, año en que Karl Neumann, si bien es cierto considera que el Handbuch es la obra capital para el estudio de la teología práctica, sin embargo plantea que habría un consenso en que «la concepción fundamental del Manual de Teología Pastoral y de la teología práctica proveniente de Rahner está superada» ${ }^{35}$.

Después de un tiempo de silencio, detectamos una segunda recepción que tiene como contexto la celebración del centenario del nacimiento de Karl Rahner. Datamos el inicio de esta nueva recepción el año 2003 con el artículo de W. Schmolly: Pastoral verantworten: Praktische Theologie ${ }^{36}$. El hito de esta segunda recepción es el simposio realizado en julio de 2004 en Innsbruck, organizado por los teólogos y teólogas de lengua alemana, con ocasión del centenario, con el título Teología con pasión pastoral: Karl Rahner y las preguntas fundamentales de la Teología Práctica. La inquietud fundamental que motiva ese encuentro es preguntarse «en qué medida los impulsos de Rahner, sin duda influyentes en el pasado, son también significativos para una teología práctica futura y si las preguntas fundamentales de la teología práctica no exigen una nueva recepción y crítica de la teología de Rahner» ${ }^{37}$.

33 S. Rautenstrauch, Entwurf einer besseren Einrichtung theologischer Schulen (Viena 1774).

34 Por ejemplo Griesel, Rütti e, incluso Schuster que fue uno de los ideadores del Handbuch junto a Rahner. Cf. F. Klostermann; R. Zerfaß (Hg.), Praktische Theologie heute (München 1974) 141-149. 150-163. 288-307. [En adelante: PThh].

35 K. Neumann, Der Praxisbezug der Theologie bei Karl Rabner (Freiburg 1980) 387. [En adelante: Der Praxisbezug].

36 W. Schmolly, «Pastoral verantworten: Praktische Theologie» en A. Batlogg et al., Der Denkweg Karl Rabners: Quellen- Entwicklungen- Perspektiven (Mainz 2003) 242-261.

37 R. FEITER et al., «Editorial» en PthI 24 (2/2004) 7. 
Abordaremos a continuación dos temas que tienen especial relevancia para la validación de la teología práctica como ciencia: la relación teoríapraxis y la autorrealización de la Iglesia. Entraremos en estos temas considerando, en un primer momento, la crítica que han suscitado para, en un segundo momento, profundizar el planteamiento de Rahner desde la perspectiva escatológica, intentando señalar -cuando sea posible- los caminos que han sido intuidos o insinuados por Rahner, pero que implican ir más allá de Rahner. En la fundamentación hacemos referencia al análisis genético de la perspectiva escatológica en la obra de Rahner, que constituye la segunda parte de nuestro trabajo de investigación doctoral, pero cuya presentación excede las posibilidades de esta ponencia. Por ello, cuando sea necesario iremos clarificando en algunos elementos de ese análisis.

\subsection{La relación teoría-praxis}

La relación entre teoría y praxis es uno de los problemas capitales asociados a la reflexión de la teología práctica. Presentamos, en primer lugar la crítica que se ha hecho al planteamiento de Rahner para, luego, esbozar una fundamentación escatológica de la praxis.

\subsubsection{La crítica al planteamiento de Rabner}

Para Norbert Mette, la teología práctica no logra diferenciarse adecuadamente de las otras ciencias teológicas por su modo de relación con la praxis. Rahner habría ampliado demasiado el círculo de problemas de la disciplina práctica, pues la teología especulativa también tiene que ver con la razón práctica. En este sentido el planteamiento de Rahner parecería no ser compatible con la definición que hace W. Kasper de la teología especulativa cuando la entiende «como reflexión sobre el origen histórico de la fe, con respecto a su posible futuro, comprensibilidad y plausibilidad, de cara a la situación espiritual actual de la Iglesia y la Humanidad ${ }^{38}$. Sería difícil pensar una eclesiología esencial válida que no prestase atención al contexto. Por ello no podría ser una tarea específica de la teología práctica transponer el conjunto de la teología del nivel de la razón teórica al nivel de la razón práctica. ${ }^{39}$

38 W. Kasper, Die methoden der Dogmatik. Einheit und Vielfalt (München 1967) 68, apud N. Mette, «Zwischen Reflexion und Entscheidung: Der Beitrag Karl Rahners zur Grundlegung der praktischen Theologie» en TrThZ 87 (1978) 26-43. 136-151; 147.

39 Cf. MetTe, Zwischen Reflexion und Entscheidung, 147; tb. «Die praktische Theologie im Verständnis Karl Rahners - in Nähe zur und in Abgrenzung von der Syste- 
En la opinión de Mette es necesaria una mayor precisión de la comprensión de una estructura de la teología práctica como teoría de la praxis, pues no basta decir que en la teología práctica es comprendida la praxis en su propia inteligibilidad con la ayuda de un análisis trascendental y, por eso, se evidenciaría la insuficiencia de la razón teórica delante de la praxis $^{40}$. En esta misma línea, Hermans y Scherer-Rath piensan que no está claro el estatuto científico de la teología práctica en Rahner, pues pareciera que esta es realizada en un primer nivel de reflexión y la teología, como ejercicio de la ciencia, debe estar siempre en un segundo nivel de reflexión. Esta diferencia de niveles no puede ser descuidada. La teología práctica, si quiere ser considerada disciplina universitaria, debe ser construida a partir de la teoría científica, lo cual exige la asunción de una distancia metódico-crítica en relación al objeto de investigación ${ }^{41}$. Si bien es cierto Rahner piensa la teología práctica en su proximidad a la praxis concreta, sin embargo no habría desarrollado una solución para el problema epistemológico. Su método trascendental es inapropiado para una construcción teórica, desde la cual pueda ser analizada la praxis vivida ${ }^{42}$.

Una de las críticas más importantes que se hace a Rahner es que una toma de decisión como la que se propone la teología práctica, que debe plasmarse en imperativos concretos de naturaleza carismática, no puede ser realizada por una ciencia. Así F. v. d. Oudenrijn dice que en el retroceso a lo indeducible radicaría la principal insuficiencia de tal teoría para la praxis, de modo que el planteamiento de Rahner sería la invitación a un total decisionismo. En el rol predominantemente profético-carismático que se atribuye a la teología práctica radicaría una confusión que descansa sobre un vicio frente a un análisis hecho sobre base empírica ${ }^{43}$. Ante esta crítica de v. d. Odernrijn, Neumann plantea que, justamente, la indeducibilidad y autonomía de la praxis es lo nuevo y lo que aporta a un avance

matischen Theologie» en PthI 24 (2/2004) 142-145; 144. [En adelante: Die praktische Theologie im Verständnis Karl Rahners].

40 Cf. MetTe, Zwischen Reflexion und Entscheidung, 149.

41 Cf. Ch. Hermans; M. Scherer-Rath, «Interdisziplinarität in der Praktischen Theologie: Epistemologische Grundfragen einer hemeneutisch-empirischen Begründung der Praktischen Theologie», PthI 24 (2/2004) 154-181; 160.

42 Cf. Hermans; Scherer-Rath, «Interdisziplinarität in der Praktischen Theologie», 161 , nota 25.

43 Cf. F. Oudenrijn, v. d. Kritische Theologie als Kritik der Theologie. Theorie und Praxis bei Karl Marx - Herausforderung der Theologie (München 1972), 182, apud METTE, Zwischen Reflexion und Entscheidung, 38. 
en la reflexión en el concepto de praxis de Rahner. La praxis no es ni adecuadamente asumida científicamente ni producida técnicamente. Praxis, en sentido aristotélico es más que poiesis, en particular, la praxis entendida cristianamente que tiene que ver siempre con amor y esperanza. Un tal concepto de praxis adquiere una dignidad teológica propia ${ }^{44}$.

Nos parece que en este intento por clarificar un estatuto particular de la praxis, que le da una dignidad y autonomía propia, hay un elemento clave en la propuesta de Rahner y que será de vital importancia para intentar clarificar los problemas planteados. Desde nuestro propio punto de vista, la novedad de Rahner radica en una fundamentación escatológica de la praxis. Es lo que intentaremos mostrar a continuación.

\subsubsection{Fundamentación escatológica de la praxis}

En el curso de nuestra investigación se ha ido asentando la convicción de que la posibilidad de una comprensión y asunción crítica de la praxis, pasa por su fundamentación escatológica. Nos parece que es desde esta perspectiva cuando la praxis eclesial se radicaliza porque se torna más libre, al reconocer su fundamento incondicional, quedando resguardado así su potencial socio-crítico. Dividimos esta fundamentación en cuatro momentos: praxis y experiencia del misterio de Dios, praxis spe formata, praxis mistagógica e inteligibilidad propia de la praxis.

\subsubsection{Praxis y experiencia del misterio de Dios}

En su fundamentación Rahner toma distancia de una concepción de la praxis como meros hechos concretos, objetivables y, en cuanto tales, posibles de ser completamente planificados y conducidos. Cuestiona una praxis entendida como mero desarrollo de las potencialidades de la materia, sea en lo que llama evolución biológica pasiva (evolucionismo), sea en la evolución cultural activa (marxismo y futuro tecnológico). No es posible determinar la praxis de un modo puramente funcional, como si fuese una factibilidad accesible o una pura productividad alcanzable. Lo fundamental, desde la perspectiva de Rahner, es que la praxis es siempre acción histórica de sujetos libres, por eso no es mera ejecución de lo planificado, aplicación de lo teórico, sino la radical apertura del ámbito de la acción, el riesgo de lo no planificado. No es algo que se pueda conducir adecuadamente desde la teoría, ni deducible desde ella simplemente, porque la po-

44 Cf. Neumann, Der Praxisbezug, 392. 
sibilidad de aquello que implica riesgo surge recién en la praxis misma ${ }^{45}$. Como acontecimiento de la libertad implica la creación de un futuro cada vez único, irrepetible. Como telón de fondo está la concepción de cada ser humano como individuum ineffabile ${ }^{46}$, evento de la autocomunicación libre y gratuita de Dios. En este sentido hay un fundamento de la libertad que es distinto de la fundamentación de la modernidad. Por ello la praxis tiene una dignidad teológica propia, goza de autonomía y no es mera esclava de la teoría. Según Neumann, para Rahner la praxis vivida tendría una prioridad sobre la reflexión, pues es siempre más rica que aquello que la reflexión puede comprender de ella ${ }^{47}$. Y esto porque la praxis tiene que ver con la relación Dios-creatura que se concreta en un llamado que Dios hace a cada ser humano, llamado único, gratuito, histórico. Llamado que tiene que ver con la plenificación de cada ser humano. Es un llamado sustentado por una relación de amor y, por ello acontece siempre en la incondicionalidad.

Con el fin de ahondar en el vínculo incondicionalidad-praxis, presentamos aquí la reflexión de Ottmar Fuchs de la Universidad de Tubinga, quien sostiene que para la fundamentación de la praxis en Rahner es clave su concepción de Dios-misterio. Esto es particularmente importante para nosotros porque considera la perspectiva escatológica en esa fundamentación y sitúa, justamente ahí, el potencial crítico de la teología práctica de Rahner. En pocas palabras, en el planteamiento de Rahner «trascendencia inagotable de Dios y diaconía radical se condicionan mutuamente» ${ }^{48}$. Para Fuchs, la pregunta por el misterio no tiene que ver solo con el ámbito del conocimiento, sino que es también un problema de la acción, pues la acción, originariamente, debe «ser entendida como la capacidad de lo incomprensible, como capacidad de ser aprehendido a través de lo siempre insometible, como proceso hacia el interior de lo inaccesible» ${ }^{49}$. Fuchs hace especial referencia a la conferencia dada por Rahner en Freiburg en

45 Cf. MetTe, Zwischen Reflexion und Entscheidung, 146.

46 Rahner utiliza el concepto que santo Tomás aplica a los ángeles para subrayar que cada ser humano es completamente único, podríamos decir: un evento escatológico, pues en el ejercicio de su libertad crea definitividad, eternidad. Cf. K. RAHner, Formale Existentialethik, 309.

47 Cf. Neumann, Praxisbezug, 250.

48 O. Fuchs, «Theologie aus der Erfahrung des 'Mysterium Dei'» en PthI, 24 (2/2004) 68-104; 76.

49 O. Fuchs, «Theologie aus der Erfahrung des 'Mysterium Dei'» 71. 
1984 (Experiencias de un teólogo católico) ${ }^{50}$, en la cual Rahner insiste en que el lenguaje sobre Dios es siempre analógico, subrayando que la incomprensibilidad de Dios permanece incluso en la visio beatifica. Al decir esto, Rahner vincula las afirmaciones teóricas al sino existencial y prohíbe así un estatus propio de la teoría delante de las experiencias, pues la esencia de la revelación cristiana es que Dios nos dice la verdad, en la medida que actúa en nosotros ${ }^{51}$. De este modo y a partir de la pregunta de Rahner: «¿Cómo puede lo incomprensible y sin nombre ser el sentido que nosotros tenemos? $\rangle^{52}$, la teología práctica debe preguntarse: ¿Cómo puede lo incomprensible y sin nombre ser la acción que nosotros hacemos? En todos los ámbitos no se nos da lo último, sino lo penúltimo y nosotros no sabemos qué significa esto en última instancia, a partir del misterio de Dios. La verificación escatológica queda pendiente en cada caso ${ }^{53}$.

El hecho de que misterio de Dios y diaconía radical crezcan proporcionalmente queda de manifiesto en la programática de la praxis de la Iglesia propuesta por Rahner, pues «es la programática de un (...) doble testimonio correspondiente: de la fe en que esta incomprensibilidad del misterio de Dios en su fundamento más profundo es amor infinito, $y$ de la esperanza en que todo amor, justicia y misericordia interhumanos, representan la praxis que les corresponde, justamente en su incondicionalidad y ausencia de expectativa para el ámbito propio. Todo está ordenado a la doxología delante de aquel Dios sorprendente, en cada situación y, con eso, a través de todas las experiencias ${ }^{54}$.

Se trataría así del ejercicio práctico de la dialéctica no hegeliana, sino la dialéctica negativa de Adorno entre misterio y pathia, donde el misterio no debe desembocar en la apatía, ni la pathia de Dios debe destruir su misterio. Es la intuición de que esta tensión se legitima de modo hermenéutico diferencial también al interior de la Trinidad, en la distinción de las personas divinas, en el rechazo del patripasionismo, así como en el rechazo

50 K. RAHNER, «Erfahrungen eines katholischen Theologen» en SW, 25, 47-57.

51 Cf. O. Fuchs, «Die Pastoral im Horizont der ,unverbrauchbaren Transzendenz Gottes' (Karl Rahner)», ThQ, 185 (2005) 268-285; 269. [En adelante: Pastoral im Horizont].

52 K. RAHNER, «Die menschliche Sinnfrage vor dem absoluten Geheimnis Gottes» en $S W, 30,209-221 ; 213$.

53 Cf. O. Fuchs, «Pastoral im Horizont», 270.

54 O. Fuchs, «Theologie aus der Erfahrung des Mysterium Dei», 85. [Cursivas del autor, negritas son nuestras]. 
de la incapacidad de sufrir de Dios en Cristo. Dios se nos ha acercado en Cristo de tal modo que él como segunda persona divina ha asumido en sí mismo todo lo que la humanidad experimenta. A partir de su encarnación con-sufre la oscuridad de lo humano en solidaridad con la humanidad pecadora y sufriente ${ }^{55}$.

En su intento de, con Rahner ir más allá de Rahner, Fuchs cree que hay que profundizar en la comprensión de la encarnación como expresión de la diferencia intratrinitaria ${ }^{56}$. Esto significa avanzar en el planteamiento de Rahner que considera que la unión hipostática es la forma insuperable del mismo misterio de Dios, el misterio expresado, la respuesta. Es necesario considerar esta entrega realizada en Cristo como la propia realidad de Dios, pues en el Evangelio el Hijo del Hombre no es solo la Palabra de Dios, que es respuesta como misterio manifestado de sí mismo, sino que en cuanto Hijo del Hombre es la pregunta, la pregunta del Cristo que está muriendo en la cruz: «Dios mío, Dios mío, ¿Por qué me has abandonado?» ${ }^{57}$. Así se establece en Dios mismo la tensión de la contradicción que grita entre $\mathrm{Pa}$ dre e Hijo en y a través del Espíritu Santo. El amor del ser humano, Hijo de Dios, a partir de su solidaridad con los sufrientes y pecadores, se torna algo conflictivo, tanto ante los seres humanos como ante Dios ${ }^{58}$.

De este modo el sufrimiento humano no es divinizado, sino que es asumido de nuevo en su distancia constitutiva de Dios en un acto libre de Dios mismo. El Hijo de Dios es la manifestación de esta asunción. Él asume el sufrimiento del ser humano justamente no hacia adentro de Dios, sino que realiza esta queja delante de Dios como Hijo del Hombre, también escatológicamente. La queja de Jesús que muere es la anticipación histórica de la solidaridad escatológica del Juez (de Mt 25), no solo con la víctima delante de los hechores, sino también con la víctima delante de Dios mismo ${ }^{59}$. "Cristo no representa sólo la respuesta amorosa al Dios incomprensible, sino también la pregunta [que se dirige] a Dios en un grito, a partir del sufrimiento del mundo» ${ }^{60}$.

55 Cf. O. Fuchs, «Theologie aus der Erfahrung des Mysterium Dei», 103-104; tb. O. Fuchs, «Pastoral im Horizont», 284.

56 Cf. O. Fuchs, «Pastoral im Horizont», 268.

57 Cf. O. Fuchs, «Pastoral im Horizont», 276.

58 Cf. O. Fuchs, «Pastoral im Horizont», 276, nota 33 y 34.

59 Cf. O. Fuchs, «Pastoral im Horizont», 277.

60 O. Fuchs, «Pastoral im Horizont», 279. 
Por ello Fuchs sostiene que «lo que en la Trinidad es el rechazo al patripasionismo, puede ser visto en la Iglesia, análogamente, como la doxología incesante, en la cual los fieles, no pasando por encima del sufrimiento y la culpa, sino a través de esas oscuras experiencias y, entonces, extendiéndose más allá de ellas, adoran y glorifican a Dios, de modo que ni la más terrible oscuridad es elevada a la condición de último, sino, en alabanza paradojal a Dios, es conducida nuevamente a aquél que tiene y tendrá la última palabra. Eso acontece principalmente en la liturgia (...) El rechazo al patripasionismo se muestra, a partir de ahí, como la expresión de la alegría esperanzada en que la historia en su forma actual no sea eterna, y en que tampoco Dios resuelva en sí eternamente la contradicción de la historia, sino en que este eón tenga un final en el que tenga que haber un nuevo comienzo eterno» ${ }^{61}$.

La pasión de Dios con el ser humano se manifiesta así no como algo frágil, sino como prueba del poder del amor y la solidaridad infinita del Dios trinitario, pues todo sufrimiento de la tierra se encuentra en Él y tiene lugar en Él ${ }^{62}$. Para Fuchs, después de Auschwitz el sufrimiento humano no puede ser puesto a salvo a partir de Dios, y eso pasa por dejar que esta diferencia en Dios mismo sea un problema. Rahner formula el sufrimiento del Señor en el modo del recuerdo del haber-sufrido [Gelittenhaben], que hay que entender de modo presente: él, con su corazón, con su existencia humana está junto a nosotros aquí y ahora. Así, por su gracia y por el Espíritu Santo su pasión adquiere nueva forma en nuestra vida, de modo que el sufrimiento de Jesús solo terminará cuando termine el sufrimiento humano. Por ello el Resucitado es el fin de los tiempos, el corazón del mundo ${ }^{63}$. En el Crucificado Dios ha asumido todo y, con eso, ha salvado todo. Ha asumido el abandono de Dios, y por ello debemos creer que Dios está cerca también allí donde pensamos que nos ha abandonado. Esto acontece sobre todo en la devoción a la humanidad de Cristo: El corazón humano del Señor que ahora es, que no pertenece más a este mundo que nos domina, que parece perdido en la lejanía de Dios, debe ser alabado, amado, a él se le debe orar. De este modo la infinitud sin límites solo se encuentra «allí donde está Jesús de Nazaret, este finitamente concreto, fortuito, que permanece eternamente» ${ }^{64}$.

\footnotetext{
61 O. Fuchs, «Theologie aus der Erfahrung des Mysterium Dei», 104.

62 Cf. O. Fuchs, «Pastoral im Horizont», 282.

63 Cf. O. Fuchs, «Pastoral im Horizont», 282-283.

64 O. FuCHS, «Pastoral im Horizont», 282.
} 
Si la gracia es la gracia de la cercanía del misterio que permanece misterio, entonces Dios se hace experimentable, pero siempre como misterio santo. Por ello la praxis del amor, e implícitamente la praxis de la Iglesia, es la condición de posibilidad de un adecuado conocimiento de Dios. Esta praxis tiene que ver con un abandono de sí mismo y con el reconocimiento de la incomprensibilidad de Dios. La acción propia nunca debe ser entendida como acceso a Dios ${ }^{65}$. Lo que expresa la perijoresis de praxis del amor y misterio de Dios es la incondicionalidad. Rahner ve en eso un llamado a la libertad, entendida como un confiarse radicalmente a la incomprensibilidad de Dios, sin reservas ni búsqueda de seguridades. Solo así la libertad se torna libre y bienaventurada. Esto tiene como consecuencia para la espiritualidad la aceptación del fracaso, pues todo anuncio de sentido, de la victoria, de la esperanza es cristiano solo en la aceptación del fracaso, en la no superación definitiva de la duda. Así el cristianismo es todo lo contrario de un opio del pueblo ${ }^{66}$. Contra una concepción superficial del testimonio cristiano, es necesario aceptar que la vida cotidiana no es pura transparencia, por ello es un error pensar que la Iglesia solo debe transmitir su verdad con claridad y transparencia, como si toda ambigüedad le fuera extraña. También sería errado pensar que la praxis de la Iglesia debe concretarse siempre en éxitos palpables. «Aquí Rahner insinúa una progresiva espiritualidad de la renuncia, que adhiere al seguimiento del Crucificado, sin permitir en eso que, con cierta violencia para con los seres humanos y para con Dios, la búsqueda del éxito sobrepase los límites ${ }^{67}$. Por ello el martirio cristiano nunca puede ser realizado para eliminar a los enemigos, sino que es siempre oferta para los otros, pero nunca imponiéndose a ellos con violencia ${ }^{68}$.

\subsubsection{Praxis spe formata}

Como consecuencia de lo recién planteado, la esperanza adquiere un lugar central a la hora de entender la praxis desde una perspectiva escatológica. Fischer cree que, así como se habla de una spes caritate formata, se puede y se tiene que hablar de una caritas spe formata. Pues tanto fe como también amor tendrían su raíz en el acto fundamental del radical aventurarse hacia la absoluta Indisponibilidad y, justamente este acto fundamental describe

\footnotetext{
65 O. Fuchs, «Pastoral im Horizont», 272.

66 O. Fuchs, «Pastoral im Horizont», 273.

67 O. Fuchs, «Pastoral im Horizont», 274.

68 Cf. O. Fuchs, «Pastoral im Horizont», 275.
} 
la esencia de la esperanza ${ }^{69}$. Y, por cuanto la praxis es fundamentalmente praxis de amor, nos parece que sería posible referirse a la praxis de la Iglesia como una praxis spe formata. La esperanza está íntimamente vinculada a la incondicionalidad, pues remite siempre al hecho personal de que el ser humano no puede, en última instancia disponer de sí, sino que se entrega a la disponibilidad de Dios. Con eso afirma el carácter de misterio de cada existencia humana como individuum ineffabile. La fe legitima la esperanza, pero no puede hacer disponible, objetivable con seguridad, el fundamento de esta legitimación. Por eso Rahner puede decir que «recién la esperanza misma establece su fundamento y, en ese sentido, lo crea» $\rangle^{70}$, porque solo ella lo agarra [ergreiff]. «La esperanza coloca, ella misma, su fundamento -ésta es su osadía, en la cual ella cree y espera, que justamente su osadía sea puesta-como-fundamento por Dios mismo, por Dios que se desvenda al ser humano como su futuro salvífico indisponible-beatificantes ${ }^{71}$.

Por ello Fischer piensa que esperanza es la palabra contemporánea clave para referirse al misterio del ser humano y que señala, al mismo tiempo su adecuada relación con Dios. Es la palabra que se refiere a la osadía de la radical indisponibilidad en dos dimensiones fundamentales del ser humano: conocimiento y $\operatorname{amor}^{72}$. «La esperanza no es algo al lado del amor, más bien es el amor aquel acto fundamental del ser humano, cuya raíz es su permanente estructura-de-esperanzas ${ }^{73}$. Fischer cree que en la teología de la esperanza de Rahner está plasmado el contenido de la indiferencia ignaciana, pues el magis entendido como condición de posibilidad del abandonarse a sí mismo en esperanza, manifiesta como gracia, como Espíritu Santo, la toma de distancia de sí mismo, por sobre sí mismo, hacia el misterio del Deus semper maior ${ }^{74}$. La lógica de la decisión de los Ejercicios, releída por Rahner en el contexto de la teología práctica está en una gran proximidad con la teología de la esperanza. De ahí que Fischer piense que esperanza sea para Rahner el lema contemporáneo para la mediación de teoría y praxis ${ }^{75}$.

69 K. FISCHER, Der Mensch als Geheimnis: Die Antropologie Karl Rahners (Freiburg 1974) 383. [En adelante: Mensch als Geheimnis].

70 K. Rahner, «Zur Theologie der Hoffnung» en $S W$ 22/2, 207-221.

71 K. Fischer, Mensch als Geheimnis, 380.

72 Cf. K. Fischer, Mensch als Geheimnis, 381. 382-383.

73 K. Fischer, Mensch als Geheimnis, 387.

74 Cf. K. Fischer, Mensch als Geheimnis, 385.

75 Cf. K. Fischer, Als großer Theologe, 46-47, apud MetTe, Zwischen Reflexion und Entscheidung, 42-43. 


\subsubsection{Praxis mistagógica}

En cuanto la praxis es siempre realizada de cara al misterio del Dios incomprensible y en cuanto es siempre conformada por la esperanza, es siempre una praxis mistagógica. Walter Schmolly cree que una mistagogía hoy día, a partir del planteamiento de Rahner, debe estar marcada por el esfuerzo de «ganar forma la esperanza en el futuro absoluto, 'Dios' -en el cual introduce en última instancia la mistagogía- en una unidad clara y comprensible con el amor al prójimo y las funciones socio-críticas de la Iglesia» ${ }^{76}$. Tres son las exigencias que este autor vincula a la necesidad de una nueva mistagogía: 1) El ser humano de hoy que vive en un mundo sin Dios, puede entender lo que se dice con la palabra Dios, recién cuando en el discurso sobre Dios es evocada su experiencia trascendental, en la que él ha tenido que vérselas siempre con el misterio santo como su futuro absoluto, más concretamente, cuando él comprende lo que es orar; 2) El ser humano de hoy debe hacer la experiencia, posible por gracia, de que es él mismo, situado en el mundo, el primer lugar del encuentro con el Dios que se comunica a sí mismo en la experiencia escatológica fundada en Cristo y su gracia; 3) La esperanza en la que introduce la mistagogía, debe manifestarse con toda claridad en su dimensión intramundana; por eso la Iglesia debe manifestar claramente que el horizonte de su praxis no es solo ella misma, sino todo ser humano y, con eso la conformación creadora [schöpferische Mitgestaltung] de un mundo que ofrece a todos los seres humanos espacio para una vida en libertad y justicia ${ }^{77}$.

\subsubsection{Inteligibilidad propia de la praxis}

Una praxis tal y como Rahner la entiende, supone una inteligibilidad propia, inteligibilidad que él considera ausente en el modo como las otras ciencias teológicas se vinculan a la praxis y, en la forma como la propia teología pastoral ha entendido su relación con la praxis; por eso propone su propia concepción de teología práctica que busca superar esta deficiencia. Así Volp piensa que, justamente cuando se adjudica a la praxis una dignidad tan alta y autónoma, es irrenunciable una enseñanza teológico-práctica «para (...) la obtención de competencia crítica para la acción

\footnotetext{
76 SChmolly, «Pastoral verantworten», 258.

77 Cf. Schmolly, «Pastoral verantworten», 260.
} 
y estrategia eclesial autónoma» ${ }^{78}$. Esta inteligibilidad propia tiene que ver con la relación de conocimiento y amor.

Ante la complejidad de vincular amor y ciencia, J.C. Scanonne nos da una pista al conectar el amor con el conocimiento sapiencial. Comentando la lógica existencial de la decisión en san Ignacio que está detrás de la teología práctica de Rahner, dice que se trata de una inteligibilidad sobrenatural, que no es el instinto, ni los sentimientos, ni el gusto. Esta ratio la entiende Rahner «como 'dejar-ser' (en libertad y amor) de la presencia (evidente y luminosa en sî) del misterio como misterio» ${ }^{79}$. Se trataría de una racionalidad sapiencial en la que se da una perijoresis de intelecto y voluntad, en la que el intelecto es suprasumido [aufhebt] en $\operatorname{amor}^{80}$. Es una inteligibilidad en el modo de la sabiduría y, en ese sentido especulativo-práctica, aunque esa inteligibilidad no pueda en la teoría, ser generada ni por la razón figurativa ni la dialéctica ${ }^{81}$. Es especulativa porque es un principio lógico y especulativo-teológico, pero lo es según la forma de la sabiduría, porque sabe más de lo que puede reflexionar y expresar teóricamente ${ }^{82}$. Este más que plenifica el conocimiento tiene que ver para Scanonne con la apertura radical hacia el otro, pues es el misterio el que lleva al conocimiento a trascenderse en un acto inaprehensible, en última instancia, en amor. En este trascenderse no pierde su esencia de conocimiento, «sino muestra solo de modo más radical su esencia como dejar-ser de la presencia del misterio, que es perceptible intelectualmente, incluso cuando oscura» ${ }^{83}$. Para Schikendantz el amor es el acto en el cual el ser humano puede aceptar la incomprensibilidad divina y, por ello, es condición de posibilidad del conocimiento. Habría, por tanto, una prioridad del amor que conoce más profundamente que el conocimiento ${ }^{84}$.

78 R. VolP, «Praktische Theologie als Theoriebildung und Kompetenzgewinnung bei F. D. Schleiermacher» en PThh, 52-64; 63.

79 J. C. Scannone, «Die Logik des Existentiellen und Geschichtlichen nach Karl Rahner» en $W T h, 82-98 ; 91$. [En adelante: «Die Logik des Existentiellen»].

80 Cf. J. C. Scannone, «Die Logik des Existentiellen», 85.

81 Cf. J. C. Scannone, «Die Logik des Existentiellen», 85, nota 6.

82 Cf. J. C. SCAnnone, «Die Logik des Existentiellen», 87, nota12.

83 J. C. SCannone, «Die Logik des Existentiellen», 92; tb. cf. C. Schickendantz, Autotrascendencia radicalizada en extrema impotencia. La comprensión de la muerte en Karl Rahner (Santiago 1999) 107. [En adelante: Autotrascendencia].

84 Cf. C. Schickendantz, Autotrascendencia, 107, nota 76. 108; tb. O. Fuchs, «Theologie aus der Erfahrung des Mysterium Dei», 81. 
Así también Neumann cree que se podría hablar de un primado del amor frente al conocimiento, y que este primado podría ser leído como primado de la praxis delante de la teoría ${ }^{85}$.

A modo de conclusión de esta reflexión sobre la relación teoría-praxis, podemos decir:

a. La teología práctica no es pura praxis, pues como ciencia, debe tomar distancia de su objeto de estudio si quiere ser una ciencia crítica.

b. La teología práctica no es simple mediadora entre teología y praxis, es decir, el instrumento que permitiría una aplicación concreta de las conclusiones de los principios generales de las ciencias dogmáticas.

c. No es solo teoría en referencia a la praxis como lo podrían ser otras ciencias teológicas.

d. Debe ser siempre teoría pues, como afirma Fuchs, lo fáctico no puede ser visto adecuadamente sin conceptualización. Si no se piensa teológico-sistemáticamente se cae en un acortamiento de la mirada y en una disminución de la capacidad crítica ${ }^{86}$.

e. La teología práctica, tal como la entiende Rahner, es teoría, pero teoría entendida como momento interno de la praxis. Ella no solo tiene como meta la praxis de la Iglesia sino que, en última instancia, proviene de ahí y solo en el actuar mismo encuentra la verificación última de ese $\operatorname{actuar}^{87}$. Cuando corresponde a la praxis una inteligibilidad que no puede ser alcanzada adecuadamente, no puede la teología práctica ser vista como surgida de la teología teórica (sistemática). Su punto de partida y su meta es la praxis ${ }^{88}$. Esto tiene como consecuencia el hecho de que sus orientaciones no pueden ser establecidas a partir de una cientificidad exacta, y por eso el imperativo del futuro nunca se puede transformar adecuadamente en un imperativo (normativo) de una ciencia refleja. Los imperativos del futuro no permanecen un pedazo de ciencia pura, sino se tornan una parte de la autorrealización histórica libre de la Igle-

85 Cf. Neumann, Der Praxisbezug, 375.

86 Cf. O. Fuchs, «Rahner systematisch-theologische und spirituell-pastorale Schriften: eine doppelte Herausforderung für die Praktischen Theologie» en PthI 24 (2/2004) 146-151; 149.

87 Cf. Metтe, Zwischen Reflexion und Entscheidung, 37.

88 Cf. MetTe, Zwischen Reflexion und Entscheidung, 138. 
sia ${ }^{89}$. Ahora bien, el primado de la praxis, según F. Schupp tendría que tener consecuencias en la determinación del status de la teoría y, por eso plantea que la prioridad de la praxis solo podría ser reconocida «si las afirmaciones teóricas y conexiones hermenéuticas fuesen aprehendidas en principio hipotéticamente, (...) para que sea concebida como teoría de la decisión, orientada al problema, que indica explícitamente sus presupuestos y, con eso, las posibilidades de su criticabilidad» ${ }^{90}$, lo cual aproxima la teología práctica a la ciencia hermenéutica.

\subsection{La autorrealización de la Iglesia}

La fundamentación de una ciencia como tal pasa por la legitimación de su objeto propio, por ello nos detenemos ahora en la fundamentación del objeto de la teología práctica, intentando profundizar desde una perspectiva escatológica. Presentamos en primer lugar, la crítica que ha suscitado el concepto autorrealización de la Iglesia y, luego, algunos elementos que pueden ayudarnos a una mejor comprensión de lo que la autorrealización significa para Rahner.

\subsubsection{Crítica al concepto autorrealización de la Iglesia}

Este objeto material de la teología práctica es uno de los elementos que ha suscitado más críticas a Rahner. Neumann, citando a Krause, va a decir que esta pregunta por el concepto autorrealización de la Iglesia es la «pregunta teológico-controversial cardinal» [kontroverstheologische Kardinalfrage] en la que se ha concentrado la discusión en torno a la teología práctica de Rahner ${ }^{91}$. Distinguimos dos dificultades fundamentales: el autocentramiento de la Iglesia y la subordinación de una eclesiología existencial a una esencial.

\subsubsection{Autocentramiento de la Iglesia}

Algunos consideran que este concepto autorrealización centra excesivamente la praxis de la Iglesia en sí misma, como si todo ella lo hiciera en última instancia por amor a sí misma, en búsqueda de la Iglesia perfecta. Esto es relevante porque dejaría en un segundo plano el encargo de la

\footnotetext{
89 Cf. METTE, Zwischen Reflexion und Entscheidung, 37-38.

90 Cf. F. Schupp, Auf dem Weg zu einer kritischen Theologie [QD 64] (Freiburg 1974), 111 117, apud MеттE. Zwischen Reflexion und Entscheidung, 149.

91 Cf. G. Krause, ThPr 2 (1967) 366-367, apud K. Neumann, Der Praxisberug, 387-388.
} 
Iglesia delante de la sociedad y el mundo ${ }^{92}$. Fürst asume la crítica de Eicher en el sentido de que el planteamiento de Rahner se reduciría a una mera autorealización del ente, dejando en un segundo plano la interacción real, el juego de acción y reacción, la lucha de influjo y resistencia del mundo real. Detrás de esto está la crítica de Eicher al concepto de identidad de la autorrealización (el principio especulativo de ser y conocer) de Rahner. La experiencia del otro exigiría interacción real y no solo autorrealización ${ }^{93}$.

Otra perspectiva del autocentramiento es la falta de referencia a Jesús. Esta crítica viene sobre todo desde el ámbito protestante. Se llega a hablar de una inversión eclesiológica en la teología práctica de Rahner ${ }^{94}$. Schuster piensa que en la teología práctica se trata del campo de actualización del Cristianismo, no solo de la Iglesia. Si bien es cierto, se puede decir que la Iglesia quiere lo mismo que quiere el Cristianismo, piensa que es una conclusión acrítica. Aunque es claro que la Iglesia debe querer siempre aquello que Jesús quería, sin embargo, la pregunta es «si la Iglesia aquí y ahora de hecho no quiere ni tiene en mente nada diferente [de lo que Jesús quería y tenía en mente]. Lo que faltaba a la antigua definición de teología práctica era una determinación de contenido más exacta de la 'realización de la Iglesia', es decir un criterio, según el cual se puede medir el legítimo deber-ser y deber-obrar de la Iglesia» ${ }^{95}$.

A partir de esa deficiencia, Schuster propone «la causa de Jesús» [die Sache Jesu] como la instancia normativa para la teología práctica ${ }^{96}$.

92 Cf. H. Schuster, «Die Praktische Theologie unter dem Anspruch der Sache Jesu» en PThh, 150-163;150; tb. G. GriESL, «Praktische Theologie als Lehre vom Selbstvollzug der Kirche» en PThh, 141-149; 148-149 y G. Biemer; P. Siller, Grundfragen der Praktischen Theologie (Mainz 1971) 136.

93 Cf. W. FürsT, «'Selbstvollzug’ der Kirche bei Karl Rahner: Philosophie- und theologiegeschichtliche Thesen», PthI 24 (2/2004) 193-196; 193. [En adelante: «Selbstvollzug der Kirche»].

94 Cf. G. SAUter, «Der Praxisbezug aller theologischen Disziplinen» en PThh, 119131,129; tb. M. KöHNLEIN, Was bringt das Sakrament? Disputation mit Karl Rahner (Göttingen 1971) 97-98.

95 H. Schuster, «Die Praktische Theologie unter dem Anspruch der Sache Jesu»en PThh, 150-163; 150.

96 Cf. H. Schuster, «Die Praktische Theologie unter dem Anspruch der Sache Jesu», 152. 


\subsubsection{Subordinación de una eclesiología existencial a una esencial}

Biemer y Siller piensan que, pese a que el planteamiento de Rahner significó un gran paso en la emancipación de la teología práctica de la dogmática y la teología moral, sin embargo, no se logró superar del todo la sumisión de la teología práctica a la dogmática «si la 'esencia de la Iglesia' determinada dogmáticamente, también determina las normas de la teología práctica» ${ }^{97}$. En la opinión de Schuster, se colocaban en el Handbuch dos criterios: por un lado, la esencia fundamental de la Iglesia con sus estructuras y funciones y, por otro lado, la situación presente en la que la Iglesia realiza su tarea; pero el problema estuvo en pensar que esa esencia fundamental fuese algo ya acabado al interior de la eclesiología esencial, como para que la teología práctica simplemente lo asumiera o, a lo más, tuviera que traducirlo simplemente a la situación presente. Fue demasiado optimista -continúa Schuster-, incluso ingenuo «esperar de una teología que trabaja dogmáticamente, principios universalmente válidos y practicables sobre una 'esencia', o bien 'esencia fundamental' de la Iglesia» ${ }^{98}$. Esto suponía, en la opinión de Rütti, una comprensión de la esencia y encargo de la Iglesia válida y aceptada universalmente, lo cual ha sido radicalmente cuestionado por las Iglesias del tercer mundo ${ }^{99}$.

V. d. Oudenrijn, analizando la categoría realización y, desde la comprensión marxista de teoría y praxis, piensa que no se verifica en el Handbuch una dialéctica real entre esencia y fenómeno en la Iglesia. De ahí surge la pregunta por el modo como una comprensión genuinamente teológica podría poner en juego la identidad de la fe en la forma histórica concreta de la Iglesia. Este autor opina que esa deficiencia dialéctica pone en evidencia que Rahner ha sostenido siempre una subordinación de la praxis a la teoría: cuando él supuso una esencia y normas esenciales que permanecen y que también norman la praxis (como sostiene Rahner contra la ética de la situación), así como cuando conoce principios dogmáticos que norman la teología práctica ${ }^{100}$.

97 Biemer; SiLler, Grundfragen der Praktischen Theologie, 135-136, apud Neumann, Der Praxisberug, 388.

98 H. Schuster, «Die Praktische Theologie unter dem Anspruch der Sache Jesu», 151.

99 Cf. L. RütTI, «Mission - Gegenstand der Praktischen Theologie odre Frage na die Gesamttheologie? Überlegungen zum Ende der kolonialen Mission» en PThh, 288$307 ; 249$.

100 Cf. Oudenrun, Kritische Theologie als Kritik der Theologie, 179-186, apud Neumann, Der Praxisberug, 389-390. 


\subsubsection{La autorrealización de la Iglesia en perspectiva escatológica}

El propio Rahner, en la segunda edición del primer volumen del Handbuch, contesta la crítica de que la autorrealización de la Iglesia representa una perspectiva autocentrada: «La palabra 'autorrealización' tiene que ser leída en su sentido formal. [Ella] no implica una 'introvertividad' de la Iglesia, como si su actuar estuviese referido finalmente a sí misma. Ella tiene que adorar a Dios, servir a la salvación del ser humano, estar ahí para el mundo. Todo esto es justamente su autorrealización, así como un ser humano se realiza a sí mismo justamente amando a Dios y, con eso, saliendo de sí mismo» ${ }^{101}$.

A su vez Neumann contesta la crítica de Schuster, pues considera que Rahner ha elaborado más diferenciadamente la esencia fundamental de la Iglesia y no lo ha hecho tan precrítica o ingenuamente como señala Schuster $^{102}$. Para Neumann autorrealización caracteriza el hecho de que existen entes, sobre todo el ser humano, que tienen que realizarse siempre de nuevo según la situación histórica. Lo mismo ocurre con la Iglesia que «no es solo factum dado, sino faciendum encomendado» ${ }^{103}$. No se trata, entonces, de que se realice externamente y manifieste una esencia dada siempre igual, sino que «significa, al contrario, que el ser humano en su autorrealización histórica 'crea su esencia como concreta', que el ser humano, diferentemente de las 'cosas', en su 'esencia', es verdaderamente alcanzado por su 'hacer' y [alcanzado] al punto de plasmar su definitividad» ${ }^{104}$.

En esa misma línea, Fürst opina que lo que está como telón de fondo de la autorrealización actual de la Iglesia es la autorrealización entendida como realización personal de la propia historicidad y temporalidad: como autorrealización existencial de la persona ${ }^{105}$.

Es interesante también la lectura que Schmolly hace del concepto de autorealización, a partir del concepto Realsymbol que Rahner trabaja en su artículo Para una teología del símbolo ${ }^{106}$ : «Si el ente es a partir de sí mismo,

101 K. RAHNER, «Ekklesiologische Grundlegung» en SW 19, 47-79; 47, nota 1. [Cursivas del autor] En esta misma línea cf. tb. MetTE, Zwischen Reflexion und Entscheidung, $30 \mathrm{y}$ Fürst, Selbstvollzug der Kirche, 194.

102 Cf. Neumann, Der Praxisbezug, 390.

103 Neumann, Der Praxisbezug, 384. [Cursivas son nuestras].

104 Neumann, Der Praxisbezug, 389. Neumann hace referencia aquí a RAHNER. «Mensch» en SW 17/2, 1199-1207; 1200-1201.

105 Cf. Fürst, Selbstvollzug der Kirche, 193-194.

106 Cf. K. Rahner, «Zur Theologie des Symbols» en SW 18, 423-457. 
simbólico, en la medida en que se realiza en sí mismo en su actualidad plural, y, en esa coincidencia de origen del otro con su origen originario, se posee a sí mismo» ${ }^{107}$, y si el Realsymbol «es la autorrealización de un ente en el otro, [autorrealización] que pertenece a la constitución esencial [de ese ente] ${ }^{108}$, entonces, autorrealización se refiere de modo formal a «la relación del fundamento de la esencia de un ente, con su necesaria expresión en otro (como diferencia en la unidad), [en otro] que pertenece a la constitución de la esencia, en ella se origina y así con ella concuerda» ${ }^{109}$. Esto significa para la Iglesia que «su esencia es dada en la unidad del fundamento de su esencia y la realización histórica de su esencia encargada a ella por Dios de modo indeducible, que al mismo tiempo determina y revela el fundamento de su esencia» ${ }^{110}$. De este modo, el concepto autorrealización contribuiría a una determinación teórico-científica clara de la relación entre eclesiología esencial y existencial ${ }^{111}$. Así es posible afirmar que la esencia de la Iglesia tiene una historia, pues se busca a sí misma recién en su historia y los principios de la autorrealización de la Iglesia aparecen, paso a paso, recién en su acontecimiento histórico ${ }^{112}$. En la fundamentación de Rahner la teología práctica debe comprender la Iglesia, esencialmente, «como una realidad teológica, que recién llega a alcanzar su esencia en una historia dispuesta por el Espíritu de Dios, cuya última ley es el misterio absoluto, 'Dios' mismo, que se comunica en Jesucristo y su gracia» ${ }^{113}$. Por su parte, Fürst valora que la comprensión más trinitaria de la Iglesia como presencia de la autocomunicación de Dios y su consecuente estructura de sacramento originario [sic], muestra de modo manifiesto un carácter capaz, al mismo tiempo, de lo escatológico y del mundo [eschatologisch-welthaften Charakter], atravesado por el principio de la esperanza ${ }^{114}$.

A partir del planteamiento de Neumann, Schmolly y Fürst, y a partir de las conclusiones de nuestra propia investigación, nos parece que el con-

107 K. RAhNer, «Zur Theologie des Symbols», 432.

108 K. Rahner, «Zur Theologie des Symbols», 435.

109 W. Schmolly, Eschatologische Hoffnung in Geschichte: Karl Rahners dogmatisches Grundverständnis der Kirche als theologische Begleitung von deren Selbstvollzug. Innsbruck: Tyrolia, 2001, 222. [En adelante: Eschatologische Hoffnung].

110 Schmolly, Eschatologische Hoffnung, 222. [Cursivas son nuestras].

111 Cf. Schmolly, Eschatologische Hoffnung, 222.

112 Cf. Schmolly, Pastoral verantworten, 245; tb. K. RAHner, Ekkelesiologische Grundlegung, 72.

113 Schmolly, Pastoral verantworten, 246.

114 Cf. Fürst, Selbstvollyug der Kirche, 195. 
cepto autorrealización de la Iglesia es valioso, sobre todo, porque contiene en sí un dinamismo escatológico. En el paso de la concepción de teología práctica de Graf: «la autoconciencia científica de la Iglesia que se autoedifica hacia el futuro» ${ }^{115}$, a la de Rahner: «la ciencia teológica que bajo el análisis científico y, precisamente, teológico de la situación concreta encada-momento de la Iglesia, desarrolla los principios (y en la medida de lo posible los concreta en imperativos), según los cuales la Iglesia en esta determinada situación actualiza su propia esencia y así realiza su actividad de salvación ${ }^{116}$, puede quedar la impresión de que se produce un debilitamiento del dinamismo escatológico que aparece, en el caso de Graf, en la expresión «hacia el futuro». Sin embargo, creemos que el dinamismo escatológico está dado en el caso de Rahner, en la «actualización de su propia esencia», o como lo formulará luego, en la autorrealización. Hay un paso de la autoconstrucción [Selbsterbaunng] a la autorrealización [Selbstvolling] que es relevante, y que diferencia la propuesta de Rahner al integrar la dimensión de plenificación de lo que se está llamado a ser, plenificación que es siempre escatológica, que pone en referencia una y otra vez a la Iglesia con su fundamento: Dios, en un proceso que acontece siempre en la historia, pero que es siempre reductio in mysterium. Nos parece que además del objeto formal dado por la condicionalidad de la situación presente, existiría otra perspectiva formal dada por el concepto autorrealización: el modo de la plenificación [in der Modus der Vollendung] ${ }^{117}$. Eso tiene como supuesto que la Iglesia es la comunidad escatológica que peregrina «en la meta hacia la meta» ${ }^{118}$. Esa sed de plenitud, la intuición de que es posible siempre más, está fundada en que esa plenitud ya le ha sido dada en la autocomunicación de Dios y, por tanto, la posee ya ahora como amor y verdad, pero siempre en esperanza. Esta condición de comunidad escatológica es lo que fundamenta la crítica y la autocrítica en la Iglesia.

Así también Rahner subraya el lugar de Jesucristo como criterio de la praxis de autorrealización, cuando dice que la historia es siempre según Cristo ${ }^{119}$ y que nos parece que hay que ensanchar a una historia según la his-

115 Cf. Midali, Teologia pastorale o pratica, 73-74.

116 K. RAHNER, «Pastoraltheologie- ein Überblick», 6-7.

117 Cf. K. Rahner, «Theologische Prinzipien der Hermeneutik eschatologischer Aussagen» en $S W 12$, 489-510; 499-500.

118 K. RAHNER, «Kirche und Parusie Christi» en SW 10, 626-640; 629.

119 Cf. K. RAHNER, «Eschatologie, theol.- wissenschaftstheoretisch» en $S W 17 / 1,220$ $225 ; 223-224$. 
toria de salvación que tiene como plenitud a Cristo. También cuando Rahner trata de la muerte de Cristo y la indiferencia ignaciana como el desde dónde de la praxis cristiana ${ }^{120}$. Por eso nos parece desproporcionada la crítica de Schuster. Lo que sí es cierto es que este vínculo con Jesucristo ha sido presentado de modo más formal por Rahner, no tematizando suficientemente el contenido de esa relación, por ejemplo, a través del recurso a las ciencias bíblicas.

\subsubsection{El sujeto de la autorrealización en perspectiva escatológica}

Tenemos la impresión de que en la reflexión sobre el sujeto de la praxis de autorrealización de la Iglesia hay un potencial crítico de gran valor para la teología práctica. Esto pasa por el vínculo uno-todos. Porque cada uno es individuum ineffabile, todos son sujetos. $\mathrm{Al}$ afirmar que todos son sujetos se quiere subrayar, sobre todo, el carácter de sujeto de los que son considerados no-sujetos. Este ser sujetos debe ser comprendido desde dos perspectivas: por un lado, todos son sujetos al interior de la Iglesia (no solo un estamento, no solo los ministros ordenados) pero, por otro lado, a la Iglesia debe importarle la autorrealización de todos los seres humanos. Se abre así un horizonte de universalidad, una meta que solo puede ser alcanzada asintóticamente y, por ello, que tensiona a la Iglesia escatológicamente, pues en su peregrinaje en la historia nunca puede descansar y decir: «Todo está cumplido».

Esto abre la posibilidad de un horizonte crítico, por cuanto acontece en cada ser humano una experiencia de Dios que es única, irrepetible y, por eso, cargada de sorpresa. Desde esa definitividad que cada uno crea como sujeto libre surgen sentidos o, simplemente preguntas que cuestionan una y otra vez nuestros sentidos establecidos. Sobre todo la experiencia de dolor de cada ser humano impedirá una síntesis final, una armonización donde todo tiene su lugar, donde todas las preguntas son respondidas. En esta línea nos parece relevante la reflexión de Schmolly cuando señala que el lugar privilegiado de la experiencia mistagógica es el propio sujeto y cuando dice que la esperanza en la que introduce la mistagogía, debe manifestarse con toda claridad en su dimensión intramundana en la conformación creadora de un mundo que ofrece a todos los seres humanos espacio para una vida en libertad y justicia. Todo esto abre con naturalidad la autorrealización de la Iglesia al horizonte del Reino de Dios.

120 Cf. K. RAHNER, «Comments by Karl Rahner on Questions raised by Avery Dulles» en SW 25, 244-247; 246. 
Lo que hemos pretendido en esta presentación es mostrar cómo la perspectiva escatológica es un horizonte de comprensión que nos desvela con mayor claridad las posibilidades de propuesta de Rahner y, por eso, su actualidad y vigencia. Nos parece que nuestro planteamiento es corroborado por las relecturas más interesantes en el contexto de la segunda recepción, que exploran justamente esta dimensión escatológica de su teología práctica.

Nuestra investigación nos ha puesto también de cara a los límites de la propuesta de Rahner, pues descubrimos en ella más una intuición que una presentación acabada de una teología pastoral. El desafío es, entonces, sobre la base de la comprensión escatológica de su teología práctica, avanzar hacia una mejor clarificación de su fundamentación, de su estructura y de su método. Nos parece que desde aquí se abren las posibilidades más promisorias para su relectura. 
Resumen: Este estudio hace eco de la poco explorada fundamentación escatológica y crítica de la teología práctica de Rahner. En primer lugar, el autor se detiene en el concepto rahneriano de teología práctica, su objeto, fines e interdisciplinariedad, mostrando, como segundo momento, en qué manera su propuesta pastoral se realiza en una perspectiva escatológica. Así, es posible avanzar hacia una mejor clarificación de la fundamentación de la teología práctica, de su estructura y de su método.

Palabras clave: Rahner, Teología Práctica, Teología Pastoral, Escatología.

Abstract: This study echoes the little explored eschatological and critical foundation of Rahner's practical theology. The author first focuses on the Raherian concept of practical theology, its purpose, goals and interdisciplinarity, showing, secondly, how its pastoral proposal is carried out from an eschatological perspective. Thus, it is possible to move towards a better clarification of the foundation, structure and method of practical theology.

Keywords: Rahner, Practical Theology, Pastoral Theology, Eschatology. 